\title{
Effects of dexmedetomidine on postoperative delirium and expression of IL-1, IL-6, and TNF- $a$ in elderly patients after hip fracture operation
}

\section{Wenchao Zhang}

Xuanwu Hospital, Capital Medical University

Tianlong Wang ( $\nabla$ Wenchaoxuanwu@163.com )

Xuanwu Hospital, Capital Medical University

\section{Geng Wang}

Beijing Jishutan Hospital

\section{Minghui Yang}

Beijing Jishutan Hospital

\section{Yan Zhou}

Beijing Jishuitan Hospital

Yi Yuan

Beijing Jishuitan Hospital

\section{Research}

Keywords: Dexmedetomidine, elderly patients, hip fracture, inflammatory factor, postoperative delirium

Posted Date: February 18th, 2020

DOI: https://doi.org/10.21203/rs.2.23900/v1

License: (1) This work is licensed under a Creative Commons Attribution 4.0 International License. Read Full License 


\section{Abstract}

Background: Postoperative delirium (POD) is a common surgical complication in elderly patients. This study investigated the effects of dexmedetomidine on POD and inflammatory factors in elderly patients with hip fracture.

Methods: The randomized, double-blind, controlled trial enrolled patients aged $\geq 65$ years who underwent operation for hip fracture in the Department of Anesthesiology in Beijing JiShuiTan Hospital from October 2016 to January 2017. The patients were divided into the DEX group and the NS group and were intravenously infused with dexmedetomidine or an equal volume of normal saline, respectively. After surgery, the incidence of delirium at postoperative day 1 (T1), day 2 (T2) and day 3 (T3) were assessed using the Ramsay score and Confusion Assessment Method (CAM) delirium scale. Interleukin (IL)-1, IL-6 and tumor necrosis factor (TNF)-a concentrations in the venous blood of the two groups of patients were detected at T0 (before surgery), T1 and T3.

Results: Data from 218 patients were analyzed with 110 patients in the DEX group and 108 in the NS group. Dexmedetomidine decreased POD incidence (18.2\% vs. $30.6 \%, \mathrm{P}=0.033)$. Compared to T0, all three inflammatory factors increased at $\mathrm{T} 1$ and then decreased at $\mathrm{T} 3$ and changes with time were significant (all $P<0.001)$. IL-6 $(P<0.001)$ and TNF- $a(P=0.003)$ levels were lower in the DEX group, but IL-1 levels were similar. The rate of adverse events was similar in the two groups.

Conclusions: Dexmedetomidine reduced the incidence of POD in elderly patients with hip fracture at an early stage, and reduced short-term IL-6 and TNF-a concentrations.

\section{Background}

Postoperative delirium (POD) is an acute confusion that occurs after an operation, characterized by disturbance of consciousness, declined ability to maintain and divert attention, and memory impairment [1]. Depending on the criteria for diagnosis, the patient population, and the surgical procedure the incidence of POD ranges from 10-70\% [2]. Alongside the unpleasant experience of POD for the patient, its occurrence also causes other problems. In hospitalized elderly patients, mental disorders such as delirium are associated with increased hospitalization stay, dementia, morbidity and mortality [3]. As the population age increases the requirement for surgical procedures in elderly patients increases. For example, the incidence of hip fractures has been increasing, and surgical treatment has become the major treatment method for elderly patients with hip fracture [4]. POD is also considered to be the most common surgical complication of elderly patients with hip fractures [5-7]. In patients undergoing hip fracture surgery, general anesthesia or subarachnoid (spinal) anesthesia show similar rates of POD [8].

The occurrence of POD is usually associated with a variety of factors, such as advanced aged, preoperative multi-system diseases, medications used in anesthesia, massive blood loss during the operation and pain stimulation [9]. One suggested mechanism for POD is overexpression of inflammatory responses due to surgical stress leading to the production of proinflammatory cytokines in the brain [10]. 
The resulting neuroinflammation can damage the brain tissue, thereby causing POD. This theory is supported by expression of peripheral inflammatory markers in delirium including C-reactive protein, tumor necrosis factor alpha (TNF-a), and interleukin (IL)-1 and IL-6 [11-14]. Therefore, decreasing the expression of inflammatory factors during the perioperative period may be of great importance for protecting the brain.

Dexmedetomidine (DEX) is a highly selective a2-receptor agonist which provides sedation, analgesia and anxiety relief [15]. As an a2-adrenergic receptor agonist DEX can also reduce the systemic inflammatory response and regulate the immune system by inhibiting the central sympathetic nervous system [16]. Moreover, it can activate anti-apoptotic signaling pathways to play protective effects on damaged cells in multiple organs including the brain $[17,18]$.

A number of studies have demonstrated that DEX can reduce the risk of delirium in elderly patients after surgery [9, 19-21], but few studies have investigated related changes in inflammatory factors. One study has shown that the mechanism of DEX may be correlated with its inhibition of inflammatory responses in addition to hypoxemia, analgesia and sleep improvement [9]. Preclinical experimental models have shown that DEX can inhibit central inflammatory responses, and reduce the production of peripheral serum TNF-a [22]. Therefore, investigation of the role of DEX in reducing the incidence of POD in elderly patients undergoing surgery for hip fracture alongside levels of inflammatory markers may provide more of an insight into the prevention of POD with DEX.

This trial aimed to investigate the effects of intraoperative sedation by intravenous application of DEX on POD of elderly patients with hip fracture. Moreover, the inflammatory responses alongside the occurrence of POD were explored by detecting expression changes of serum TNF- $a$, IL- 1 and IL- 6 at different time points after surgery.

\section{Methods}

\section{Patients}

This prospective, randomized, double-blind, controlled trial was approved by the Institutional Review Board of the Beijing Jishuitan Hospital (Approval No.: 201606-09), and the patients signed written informed consent. The registration number of the clinical experiment was: ChiCTR-OON-16008691. Our study enrolled elderly patients with hip fracture treated in the Department of Anesthesiology, Beijing Jishuitan Hospital from October 2016 to January 2017.

The inclusion criteria were: 1) patients with hip fracture undergoing operation; 2) patients aged 65-90 years; 3) American Society of Anesthesiologists (ASA) physical status scale grade I-III [23].

The exclusion criteria were: 1) patients with a history of psychosis or of long-term psychotropic medication use (dementia, schizophrenia), history of chronic analgesic use or history of alcohol abuse; 2 ) patients with preoperative mini-mental state examination (MMSE [24]) score of $\leq 23$, which was assessed 
at 1 day before surgery (T0);3) patients who were illiterate; 4) patients with hearing and visual impairments as well as those who had any cerebrovascular accidents such as stroke or transient ischemic attack (TIA) within 3 months; 5) patients with severe infection; 6) patients with communication barriers who could not complete the cognitive function test.

A computer-generated random number scheme was used to assign the patients into two groups, the DEX group $(n=110)$ and the normal saline (NS) group $(n=108)$.

The patients and researchers (responsible for data recording and analysis, Confusion Assessment Method (CAM [25]) delirium scale assessment and POD diagnosis) were blinded to the groups, but the anesthesiologists knew about the medication of the patients.

Anesthetic management

Patients in both groups underwent proximal femoral nail anti-rotation (PFNA), cannulated screw fixation, hemi-arthroplasty, or total hip arthroplasty (THA). The surgical procedures were perfomred according to standard procedures. The operations for all patients were performed by the same attending physicians (vice-senior physicians) with 5-10 years of surgical experience.

The anesthetic management was the same for both groups of patients except for the administration of DEX. Electrocardiogram (ECG), arterial blood pressure (ABP), heart rate (HR) and pulse oxygen saturation $\left(\mathrm{SpO}_{2}\right)$ results were monitored after the patients entered the operating room. The patients were in supine position, and a portable ultrasound unit (WISONIC CLOVER 60VET) with a 4-12 MHz Convex array probe was used to identify the femoral artery, femoral nerve and fascia. The puncture point was at the point 2 $\mathrm{cm}$ towards the caudal side from the point of $1 / 3$ length externally along the line between the anterior superior iliac spine and the pubic tubercle. The in-plane needle approach was applied. After the nerve block needle reached the fascia iliaca compartment, $30 \mathrm{ml}$ of $0.4 \%$ ropivacaine was injected. Twenty minutes after nerve block, the patients were assisted by 3 physicians into the spinal anesthesia position, thus the diseased side was on the top, and a subarachnoid block was performed in the lateral position. The puncture point was in the L3-4 gap, and $12 \mathrm{mg}$ of 0.5 ropivacaine was injected through the subarachnoid space after the puncture was successful; otherwise, puncture was performed between L2-3. General anesthesia was performed if spinal anesthesia failed. Etomidate $(0.3 \mathrm{mg} / \mathrm{kg})$ was used in general anesthesia, sufentanil $(0.1 \mu \mathrm{g} / \mathrm{kg})$ was used for induction, and rocuronium $(0.6 \mathrm{mg} / \mathrm{kg})$ was also applied. After successful intubation, a ventilator was connected to control breathing. Propofol was continuously pumped in and sevoflurane anesthesia was inhaled during the operation to maintain the anesthesia. Anesthesia depth was maintained by continuous pumping in $2 \%$ sevoflurane and propofol. Dexmedetomidine $(0.5 \mu \mathrm{g} / \mathrm{kg} / \mathrm{h})$ was intravenously infused 30 minutes before the start of anesthesia in the DEX group, and was continuously infused at $0.3 \mu \mathrm{g} / \mathrm{kg} / \mathrm{h}$ during the operation. The same volume of normal saline was administrated for the NS group. The medication was discontinued 30 min before the end of surgery. Self-controlled analgesia was performed by administrating patient controlled intravenous analgesia (PCIA), and sufentanil combined with flurbiprofen ester immediately after the operation. 
Demographics and medical history of the patients in both groups were recorded, including gender, age, ASA grade, body mass index (BMI), education level, type of fracture, type of anesthesia, surgical procedure, operation time and comorbidities.

\section{Primary endpoint}

The primary endpoint was the incidence of POD, which was assessed using the Chinese version of the Richmond Agitation Sedation Scale (RASS) [26] and the CAM Scale at the first postoperative day (T1), the second postoperative day (T2), and the third postoperative day (T3).

CAM assessment was performed for patients with RASS of >-4. The diagnostic criteria for positive CAM [25] was as follows: (1) acute onset, and fluctuation of the disease condition; (2) distracted attention; (3) thinking disorder; (4) changes of consciousness. If patients showed characteristics of points 1 and 2 and any one of 3 and 4 , then CAM was considered positive.

Patients with positive CAM at any time point during the follow-up period were diagnosed by a psychiatrist or psychologist based on the Diagnostic and Statistical Manual of Mental Disorders 5 (DSM-5) [27]. Delirium was diagnosed if the following 5 items were satisfied based on DSM-5: (1) Attention deficit disorder; (2) acute onset and repeated changes of symptoms; (3) combined with cognitive impairment; (4) standards (1) and (3) cannot be explained by existing neurological diseases, and arousal disorder was excluded; (5) it can be seen from medical history, physical examination or laboratory tests that it was direct physiological consequences of general physical conditions.

Two trained individuals in our department conducted the assessment of POD, and they were also blinded to the grouping.

\section{Secondary endpoints}

Biochemical tests

Five $\mathrm{ml}$ of venous blood from the side without infusion was extracted at 9:00 in the morning at T0, T1 and T3, and was centrifuged at $4000 \mathrm{r} / \mathrm{min}$ for $10 \mathrm{~min}$. The serum was separated and stored at $-80{ }^{\circ} \mathrm{C}$. Before assay, all samples were thawed to room temperature and mixed by gentle swirling or inversion. All sera were assayed on the same day to avoid inter-assay variation. Plasma IL-1, IL- 6 and TNF-a levels were measured by an enzyme-linked immunosorbent assay (ELISA) kit from Bender MedSystems (GmbH, Vienna, Austria). The ELISA methods were basically the same involving a two antibody sandwich ABCELISA method. The first antibody was anti-human TNF-a, IL-1, or IL-6 monoclonal antibody and the second antibody was a monoclonal antibody containing biotin. Enzyme-labelled streptavidin was bound to the biotin, o-phenylenediamine was added, and the mixture turned yellow. At this time, sulfuric acid was added, and the color became darker. The optical density (OD) value was measured at $492 \mathrm{~nm}$ and 
the concentrations of TNF-a, IL-1, and IL- 6 were proportional to OD value. Concentration of TNF-a, IL-1, or IL-6 in the specimen were calculated by plotting a standard curve.

The antibodies used in this procedure have no known cross-reactivities with other cytokines. The lowest detectable limits of IL-1 $\beta$, IL-6, and TNF-a, were $1.5 \mathrm{pg} / \mathrm{mL}, 5 \mathrm{pg} / \mathrm{mL}$, and $1.7 \mathrm{pg} / \mathrm{mL}$, respectively.

NRS scores

Postoperative pain was assessed at T1, T2 and T3 according to a numeric rating scale (NRS), where "0" means no pain at all and " 10 " means extremely serious pain. Patients with a NRS score of $\geq 4$ after the operation were intravenously injected with $40 \mathrm{mg}$ of parecoxib sodium for analgesia in both groups.

Adverse events

Invasive artery blood pressure (systolic (SBP), diastolic (DBP), mean arterial pressure (MAP)), HR, ECG, and $\mathrm{SpO}_{2}$ were routinely monitored during the operation. The number of intraoperative adverse reactions including hypertension, hypotension, bradycardia and tachycardia were recorded. Tachycardia was defined as heart rate $>100$ bpm; bradycardia was defined as heart rate $<60$ bpm; hypertension was defined as systolic pressure $>160 \mathrm{mmHg}$ or $20 \%$ of baseline; hypotension was defined as systolic pressure $<90 \mathrm{mmHg}$ or $20 \%$ of baseline. Patients with bradycardia were administrated with $0.1-0.3 \mathrm{mg}$ of atropine, and patients with hypotension were intravenously injected with 4 ug of norepinephrine or intravenously infused at $200 \mathrm{ug} / \mathrm{h}$.

Statistical analysis

The incidence of postoperative delirium in a comparable patient population of a previous study was $28 \%$ [4]. We; therefore, assumed that the incidence of delirium would be reduced by one third in the DEX group in this trial. With significance set at 0.05 and power set at $80 \%$, the sample size required to detect differences was 196, calculated with Pass 11.0 software (NCSS, LLC. Kaysville, Utah, USA). Taking into account a loss-to-follow-up rate of about $6 \%$, we planned to enroll 208 patients.

Continuous data with normal distribution were expressed as means \pm standard deviation (SD) and analyzed by independent $t$ test, and those with non-normal distribution were expressed as medians (interquartile range) and analyzed by the Mann-Whitney u test. Categorical variables were expressed as frequencies and percentages and analyzed with chi-square analysis or Fisher exact test. Repeated measurement analysis of variance was used to analyse levels of inflammatory factors. Statistical analyses were done on SPSS Statistics for Windows (version 22.0; IBM Corp., Armonk, New York, USA) with two-tailed tests wherever appropriate and $\mathrm{P}<0.05$ were considered as statistical significant. The Clinical Research Ethics Committee from Beijing Jishuitan Hospital was involved in overseeing the data.

\section{Results}

Patient inclusion 
A total of 402 patients were assessed for eligibility. 162 patients were excluded based on the inclusion criteria established for this trial or because they declined to participate. The remaining 240 patients were randomly assigned to the DEX group or NS group. Among these patients, data for 22 patients were not analyzed due to transferring to other hospitals after operation $(n=14,6$ in the DEX group and 8 in the NS group) or they withdrew consent ( $n=8,4$ in each group). The remaining 218 patients were included in the data analysis, 110 in the DEX group and 108 in the NS group (Figure 1).

Demographics and medical history

There were no significant differences between the two groups in demographics including age, gender, BMI, education level, ASA grade, comorbidities, and type of fracture, anesthesia, surgical procedure, and operation time (all P>0.05) (Table 1).

Incidence of POD and NRS scores

There was a lower incidence of POD at T1 in the DEX group than in the NS group (14.55\% vs. $26.85 \%$, $\mathrm{P}=0.025)$ and in total POD in the DEX group than in the NS group ( $18.18 \%$ vs. $30.56 \%, P=0.033)$. However, the POD incidence decreased in both groups and was similar between them at T2 (3.64\% vs. $4.63 \%$, $\mathrm{P}=0.747)$ and T3 (1.82\% vs. $2.78 \%, \mathrm{P}=0.682)$. NRS scores were similar between the two groups at T1, T2 and T3 (all P>0.05) (Table 2).

Inflammatory markers

The changes in inflammatory markers in the two groups are shown in Figure 2 and Table 3 . In both groups, all three markers showed an initial increase at T1 and then decreased at T3. The IL-1 serum concentration changes were similar in both groups and remained above the T0 level at T1 and T3 while both groups showed a significant difference with time $(P<0.001)$. The serum IL-6 concentration was increased at T1 and T3 compared to T0. However, the DEX group showed lower serum IL-6 concentrations than the NS group at T1 and T3 $(P<0.001)$.

In both groups, serum TNF-a concentration increased at T1 and T3. However, the DEX group showed lower TNF-a concentrations than the NS group at T1 and T3 ( $\mathrm{P}=0.003)$ (Table3).

Adverse events

In total 102 adverse events were recorded 44 in the NS group and 58 in the DEX group. These included tachycardia, bradycardia, hypertension, and hypotension the rates were similar in the two groups (all $\mathrm{P}>0.05$ ) (Table 4).

\section{Discussion}

The aim of this trial was to investigate the effects of DEX on POD and level of inflammatory factors in elderly patients undergoing surgery for hip fractures. Data from 218 patients were analyzed with 110 
patients in the DEX group and 108 in the NS group. DEX decreased POD incidence to $18.2 \%$ from $30.6 \%$ in the NS group. IL-1, IL-6, and TNF-a serum levels all increased one day after surgery and then decreased on the third day after surgery. The DEX group IL- 6 and TNF-a levels were lower than in the NS group, but IL-1 levels were similar. The rate of adverse events was similar. These results show that DEX reduced the incidence of POD in elderly patients with hip fracture at an early stage and reduced the short-term increases in IL-6 and TNF-a concentrations post-surgery.

Our results suggest that intravenous administration of dexmedetomidine during surgery significantly reduces the incidence of delirium in elderly hip fracture. This result agrees with numerous other studies that have also shown reduced rates of POD in elderly surgical patients [9, 19-21]. However, by monitoring the serum inflammatory cytokines IL-1, IL-6, TNF- $a$ of the patients in this trial at different time points, we also aimed to investigate the expression of postoperative inflammatory cytokines with DEX administration and the occurrence of POD. Fewer studies have undertaken investigation of cytokines during DEX treatment to prevent POD. A study of 354 patients $>65$ years of age undergoing laparoscopic major non-cardiac surgery under general anesthesia found that POD was reduced and IL- 6 levels were significantly lower at $1 \mathrm{~h}$ and $24 \mathrm{~h}$ when DEX was administered as a bolus before surgery and by infusion from induction of anesthesia to the end of surgery [28]. For 40 patients undergoing robot-assisted laparoscopic radical cystectomy and ileal conduit diversion, the levels of TNF-a, NSE and IL-6 in the DEX group were significantly lower than in the control group one- and five-days after operation [29]. In young patients combined usage of DEX and sufentanil was investigated to treat POD after general anesthesia [30]. The results showed that levels of IL-6, and TNF-a decreased $1 \mathrm{~h}$ to $8 \mathrm{~h}$ after surgery. When the results of these studies and our results are taken together there is a suggestion that there might be a relationship between inflammatory factors and POD. In our study on the 1 st day after surgery, the levels of IL-1, IL-6, TNF-a were relatively high, and the incidence of POD was also relatively high (26.85\% and $14.55 \%)$. On the 3rd day, level of IL-1, IL-6, TNF-a were lower, and incidence of POD dropped to $2.78 \%$ and $1.82 \%$. However, further studies are needed to investigate whether there is a direct correlation between POD and cytokine levels.

The pathological mechanism of POD is still unclear, and it may be caused by multiple factors. However, inflammatory responses and neuroinflammation seem to be the main cause. Therefore, DEX, as areceptor agonist, can prevent the occurrence of POD. Elderly patients with hip fractures often have agitation during surgery due to trauma and the discomfort caused by their positioning, especially patients receiving intramedullary needle fixation. The sedative effect of DEX can reduce the patient's agitation during the operation. There is some concern that application of DEX has many potential side effects, including elevated blood pressure, decreased heart rated and inhibition of cardiac conduction system. For elderly patients with hip fracture, it has been suggested that DEX should be administered in the lowest possible doses for the shortest possible time. In our experiment, the initial dose was 30 ug for elderly patients with hip fracture, the infusing dose was $0.2 \mathrm{ug} / \mathrm{kg} / \mathrm{h}$, and medication was discontinued $10 \mathrm{~min}$ before the end of the operation. In respect to safety, our data revealed that DEX -induced bradycardia and hypotension were not significantly increased, possibly because of the very low doses that were used.Nevertheless, the hemodynamic-stabilizing property of DEX may be beneficial. Stable heart rate and 
mean arterial pressure can reduce myocardial oxygen consumption, thus this property contributes to the decrease in the incidence of cardiac events for high-risk patients [31].

There were several limitations in this study. Firstly, this was a single centre study and there might be selection bias. Secondly, inflammatory indicators were measured at only three time points, but blood specimens were not collected before anesthesia and immediately after anesthesia. Thirdly, the follow-up time was short (only 3 days after surgery), which was mainly because hospitalization of the patients was short, and blood samples could not be obtained effectively after discharge.

\section{Conclusion}

Dexmedetomidine reduced the incidence of POD of elderly patients after surgery for hip fractures. Dexmedetomidine also significantly alleviated the increase in short-term IL-6 and TNF-a levels. Dexmedetomidine may benefit patients by reducing the incidence of early POD and offering a better short-term recovery for elderly patients receiving hip arthroplasty.

\section{List Of Abbreviations}

POD: Postoperative delirium; CAM: Confusion Assessment Method; IL: Interleukin; IL-6TNF and tumor necrosis factor; ASA: Anesthesiologists; TIA: transient ischemic attack; PFNA: proximal femoral nail antirotation; THA: total hip arthroplasty; ECG: Electrocardiogram; ABP: arterial blood pressure; HR: heart rate; PCIA: patient controlled intravenous analgesia; BMI: body mass index; RASS: Richmond Agitation Sedation Scale; ELISA: enzyme-linked immunosorbent assay; OD: optical density.

\section{Declarations}

Ethics approval and consent to participate

This prospective, randomized, double-blind, controlled trial was approved by the Institutional Review Board of the Beijing Jishuitan Hospital (Approval No.: 201606-09), and the patients signed written informed consent.

Consent for publication

Not applicable

Availability of data and materials

The datasets used and/or analysed during the current study are available from the corresponding author on reasonable request.

Competing interests 
The authors declare that they have no competing interests

Funding

This study was supported by Beijing Jishuitan Hospital Nova Program [no. xkxx201619], Capital's Funds for Health Improvement and Research [2018-1-2071], and Beijing Municipal Administration of Hospitals Clinical Medicine Development of Special Funding Support [ZYLX201813].

Authors' contributions

WZ and TW contributed conception and design of the study; WZ and GW organized the database; WZ performed the statistical analysis; MY, WZ and TW wrote the first draft of the manuscript; TW, YZ, YY, and MY wrote sections of the manuscript. All authors contributed to manuscript revision, read and approved the submitted version.

Acknowledgements

The authors thank all the medical staff in the Department of Anesthesiology in Xuanwu Hospital, Capital Medical University, all the medical staff in the Department of Anesthesiology, Traumatic Orthopedics and Laboratory in Beijing Jishuitan Hospital for their support to this clinical trial.

\section{References}

1. Numan T, van den Boogaard M, Kamper AM, Rood PJT, Peelen LM, Slooter AJC, et al. Recognition of Delirium in Postoperative Elderly Patients: A Multicenter Study. J Am Geriatr Soc. 2017;65:1932-8. doi: $10.1111 /$ jgs. 14933

2. Schenning KJ, Deiner SG. Postoperative Delirium in the Geriatric Patient. Anesthesiol Clin. 2015;33:505-16. doi: 10.1016/j.anclin.2015.05.007

3. Krogseth M, Wyller TB, Engedal K, Julieb $\emptyset$ V. Delirium is an important predictor of incident dementia among elderly hip fracture patients. Dementia \& Geriatric Cognitive Disorders. 2011;31:63-70. doi:

4. Robinson TN, Raeburn CD, Tran ZV, Angles EM, Brenner LA, Moss M. Postoperative delirium in the elderly: risk factors and outcomes. Annals of Surgery. 2009;249:173-8. doi:

5. Yang Y, Zhao X, Dong T, Yang Z, Zhang Q, Zhang Y. Risk factors for postoperative delirium following hip fracture repair in elderly patients: a systematic review and meta-analysis. Aging Clinical \& Experimental Research. 2016;29:1-12. doi:

6. Wang NY, Hirao A, Sieber F. Association between intraoperative blood pressure and postoperative delirium in elderly hip fracture patients. Plos One. 2015;10:e0123892. doi:

7. Bruce AJ, Ritchie CW, Blizard R, Lai R, Raven P. The incidence of delirium associated with orthopedic surgery: a meta-analytic review. International Psychogeriatrics. 2007;19:197-214. doi:

8. Tzimas P, Samara E, Petrou A, Korompilias A, Chalkias A, Papadopoulos G. The influence of anesthetic techniques on postoperative cognitive function in elderly patients undergoing hip fracture 
surgery: General vs spinal anesthesia. Injury. 2018;49:2221-6. doi: 10.1016/j.injury.2018.09.023

9. Su X, Meng ZT, Wu XH, Cui F, Li HL, Wang DX, et al. Dexmedetomidine for prevention of delirium in elderly patients after non-cardiac surgery: a randomised, double-blind, placebo-controlled trial. Lancet. 2016;388:1893. doi:

10. Spiegel DR, Chen V. A case of postoperative cognitive decline, with a highly elevated C- reactive protein, status post left ventricular assist device insertion: a review of the neuroinflammatory hypothesis of delirium. Innov Clin Neurosci. 2012;9:35-41. doi:

11. Capri M, Yani SL, Chattat R, Fortuna D, Bucci L, Lanzarini C, et al. Pre-Operative, High-IL-6 Blood Level is a Risk Factor of Post-Operative Delirium Onset in Old Patients. Front Endocrinol (Lausanne). 2014;5:173. doi: 10.3389/fendo.2014.00173

12. Cape E, Hall RJ, van Munster BC, de Vries A, Howie SE, Pearson A, et al. Cerebrospinal fluid markers of neuroinflammation in delirium: a role for interleukin-1beta in delirium after hip fracture. $\mathrm{J}$ Psychosom Res. 2014;77:219-25. doi: 10.1016/j.jpsychores.2014.06.014

13. Cerejeira J, Lagarto L, Mukaetova-Ladinska EB. The immunology of delirium. Neuroimmunomodulation. 2014;21:72-8. doi: 10.1159/000356526

14. Gool WAV, Beek DVD, Eikelenboom P. Systemic infection and delirium: when cytokines and acetylcholine collide. Lancet. 2010;375:773-5. doi:

15. Keating GM. Dexmedetomidine: A Review of Its Use for Sedation in the Intensive Care Setting. Drugs. 2015;75:1119-30. doi: 10.1007/s40265-015-0419-5

16. Davy A, Fessler J, Fischler M, M LEG. Dexmedetomidine and general anesthesia: a narrative literature review of its major indications for use in adults undergoing non-cardiac surgery. Minerva Anestesiol. 2017;83:1294-308. doi: 10.23736/S0375-9393.17.12040-7

17. Wang D, Xu X, Wu YG, Lyu L, Zhou ZW, Zhang JN. Dexmedetomidine attenuates traumatic brain injury: action pathway and mechanisms. Neural Regen Res. 2018;13:819-26. doi: 10.4103/16735374.232529

18. Akpinar O, Naziroglu M, Akpinar H. Different doses of dexmedetomidine reduce plasma cytokine production, brain oxidative injury, PARP and caspase expression levels but increase liver oxidative toxicity in cerebral ischemia-induced rats. Brain Res Bull. 2017;130:1-9. doi: 10.1016/j.brainresbull.2016.12.005

19. Zeng H, Li Z, He J, Fu W. Dexmedetomidine for the prevention of postoperative delirium in elderly patients undergoing noncardiac surgery: A meta-analysis of randomized controlled trials. PLoS One. 2019;14:e0218088. doi: 10.1371/journal.pone.0218088

20. Deiner S, Luo X, Lin HM, Sessler DI, Saager L, Sieber FE, et al. Intraoperative Infusion of Dexmedetomidine for Prevention of Postoperative Delirium and Cognitive Dysfunction in Elderly Patients Undergoing Major Elective Noncardiac Surgery: A Randomized Clinical Trial. JAMA Surg. 2017;152:e171505. doi: 10.1001/jamasurg.2017.1505

21. Karren $E A$, King $A B$, Hughes $C G$. Dexmedetomidine for prevention of delirium in elderly patients after non-cardiac surgery. J Thorac Dis. 2016;8:E1759-E62. doi: 10.21037/jtd.2016.12.56 
22. Xiang H, Hu B, Li Z, Li J. Dexmedetomidine controls systemic cytokine levels through the cholinergic anti-inflammatory pathway. Inflammation. 2014;37:1763-70. doi: 10.1007/s10753-014-9906-1

23. Sankar A, Johnson SR, Beattie WS, Tait G, Wijeysundera DN. Reliability of the American Society of Anesthesiologists physical status scale in clinical practice. Br J Anaesth. 2014;113:424-32. doi: $10.1093 / \mathrm{bja} / \mathrm{aeu} 100$

24. Li H, Jia J, Yang Z. Mini-Mental State Examination in Elderly Chinese: A Population-Based Normative Study. J Alzheimers Dis. 2016;53:487-96. doi: 10.3233/JAD-160119

25. Inouye SK, van Dyck CH, Alessi CA, Balkin S, Siegal AP, Horwitz RI. Clarifying confusion: the confusion assessment method. A new method for detection of delirium. Ann Intern Med. 1990;113:941-8. doi: 10.7326/0003-4819-113-12-941

26. Chester JG, Beth Harrington M, Rudolph JL, Group VADW. Serial administration of a modified Richmond Agitation and Sedation Scale for delirium screening. J Hosp Med. 2012;7:450-3. doi: 10.1002/jhm.1003

27. Association AP. Diagnostic and statistical manual of mental disorders, Fifth edition 5ed. Arlington, VA, USA: American Psychiatric Association; 2013.

28. Lee $\mathrm{C}$, Lee $\mathrm{CH}$, Lee $\mathrm{G}$, Lee $\mathrm{M}, \mathrm{Hwang} J$. The effect of the timing and dose of dexmedetomidine on postoperative delirium in elderly patients after laparoscopic major non-cardiac surgery: A double blind randomized controlled study. J Clin Anesth. 2018;47:27-32. doi: 10.1016/j.jclinane.2018.03.007

29. Ding L, Zhang H, Mi W, Wang T, He Y, Zhang X, et al. Effects of dexmedetomidine on anesthesia recovery period and postoperative cognitive function of patients after robot-assisted laparoscopic radical cystectomy. Int J Clin Exp Med. 2015;8:11388-95. doi:

30. Liu L, Yuan Q, Wang Y, Gao W, Hou J, Wu Y, et al. Effects of Dexmedetomidine Combined with Sufentanil on Postoperative Delirium in Young Patients After General Anesthesia. Med Sci Monit. 2018;24:8925-32. doi: 10.12659/MSM.911366

31. Chalikonda SA. Alpha2-adrenergic agonists and their role in the prevention of perioperative adverse cardiac events. Aana J. 2009;77:103-8. doi:

\section{Tables}

Table 1. Demographics and medical history of patients in both groups 
Characteristics

\section{Age (years)}

Gender (male)

BMI $\left(\mathrm{kg} / \mathrm{m}^{2}\right)$

Education level (years)

$<5$

$5-8$

$>8$

ASA grade

I

II

III

Operation time (min)
NS group $(\mathrm{N}=108) \quad$ DEX group $(\mathrm{N}=110) \quad$ P-value

$79.14 \pm 6.79$

$78.46 \pm 6.42$

0.451

34 (31.48\%)

34 (30.91\%)

1.000

$23.40 \pm 2.20$

$23.95 \pm 2.84$

0.111

0.731

$80(74.07 \%)$

79 (71.82\%)

$26(24.07 \%)$

$30(27.27 \%)$

$2(1.85 \%)$

1 (0.91\%)

0.622

9 (8.33\%)

7 (6.36\%)

76 (70.37\%)

74 (67.27\%)

$23(21.30 \%)$

29 (26.36\%)

60.00

(40.00

70.00)

Comorbidities

Hypertension

57 (52.78\%)

58 (52.73\%)

1.000

Diabetes

21 (19.44\%)

$30(27.27 \%)$

0.172 
Heart failure

Lung infection

Chronic bronchitis

Atrial fibrillation

Arrhythmia

Anesthesia

Spinal anesthesia

Combined

anesthesia

General anesthesia

Type of fracture

Femoral neck fractures

Intertrochanteric fracture

Subtrochanteric fractures

Surgical procedure

PFNA

Cannulated screw fixation

Hemi-arthroplasty
$9(8.33 \%)$

$1(0.93 \%)$

$9(8.33 \%)$

$5(4.63 \%)$

$3(2.78 \%)$

0.399

$87(80.56 \%)$

$9(8.18 \%)$

7 (6.36\%)

0.575

5 (4.55\%)

0.739

0.223

1.000

93 (84.55\%) spinal-epidural 17 (15.74\%)
$16(14.55 \%)$

$4(3.70 \%)$

$1(0.91 \%)$

0.582

$52(48.15 \%)$

$45(40.91 \%)$

$54(50.00 \%)$

$62(56.36 \%)$

$2(1.85 \%)$

$3(2.73 \%)$

0.454

$54(50.00 \%)$

$50(45.45 \%)$

29 (26.85\%)

$24(21.82 \%)$

$14(12.96 \%)$

$21(19.09 \%)$ 
Abbreviations: $\mathrm{NS}=$ normal saline, $\mathrm{DEX}=$ dexmedetomidine, $\mathrm{BMI}=$ body mass index, $\mathrm{ASA}=$ American Society of Anesthesiologists physical status scale grade, PFNA= proximal femoral nail anti-rotation; THA= total hip arthroplasty.

Table 2. Incidence of POD and NRS scores after surgery

\begin{tabular}{llll}
\hline & NS group (N=108) & DEX group (N=110) & P-value \\
\hline POD & & & \\
T1 & $29(26.85 \%)$ & $16(14.55 \%)$ & 0.025 \\
\hline T2 & $5(4.63 \%)$ & $4(3.64 \%)$ & 0.747 \\
\hline T3 & $3(2.78 \%)$ & $2(1.82 \%)$ & 0.682 \\
\hline Total & $33(30.56 \%)$ & $20(18.18 \%)$ & 0.033 \\
\hline NRS & & & \\
\hline T1 & $2.00(2.00-3.00)$ & $2.00(2.00-3.00)$ & 0.748 \\
\hline T2 & $1.00(1.00-1.00)$ & $1.00(1.00-1.00)$ & 0.862 \\
\hline T3 & $1.00(1.00-1.00)$ & $1.00(1.00-1.00)$ & 0.509 \\
\hline
\end{tabular}

Abbreviations: $\mathrm{POD}=$ postoperative delirium, $\mathrm{NS}=$ normal saline, $\mathrm{DEX}=$ dexmedetomidine, $\mathrm{NRS}=$ numeric rating scale, $\mathrm{T} 1=1$ day after surgery, $\mathrm{T} 2=2$ days after surgery, $\mathrm{T} 3=3$ days 
after surgery.

Table 3. Levels of IL-1, IL-6 and TNF- $\alpha$

\begin{tabular}{|c|c|c|c|c|}
\hline $\begin{array}{l}\mathrm{NS} \quad \text { group } \\
(\mathrm{N}=108)\end{array}$ & $\begin{array}{ll}\text { DEX } & \text { group } \\
(\mathrm{N}=110) & \end{array}$ & $\begin{array}{l}\mathrm{F} \text { (group)/P- } \\
\text { value }\end{array}$ & $\begin{array}{l}\mathrm{F} \text { (time)/P- } \\
\text { value }\end{array}$ & $\begin{array}{l}\mathrm{F} \text { (group*time)/P- } \\
\text { value }\end{array}$ \\
\hline
\end{tabular}

IL-1

T0 $5.19 \pm 0.67$

$5.10 \pm 0.71$

$F=0.075$

$F=1226.917 \quad F=0.532$

$\mathrm{T} 1 \quad 10.88 \pm 1.51$

$11.05 \pm 1.87$

$P=0.784$

$P<0.001$

$P=0.588$

T3 $7.99 \pm 1.27$

$7.99 \pm 1.31$

IL-6

T0 $22.94 \pm 5.39$

$23.71 \pm 5.83$

$F=36.520$

$F=2804.130$

$F=36.593$

T1 $105.15 \pm 16.18$

$89.04 \pm 10.83$

$P<0.001$

$P<0.001$

$P<0.001$

Т3 $63.26 \pm 22.08 \quad 55.36 \pm 16.31$

TNF-

$\alpha$

T0 $\quad 4.35 \pm 0.73$

$4.40 \pm 0.78$

$F=7.980$

$F=72.608$

$F=5.340$

$\mathrm{T} 1 \quad 5.37 \pm 0.85$

$4.93 \pm 0.91$

$P=0.003$

$P<0.001$

$P=0.024$

T3 $4.47 \pm 0.89$

$4.19 \pm 0.92$ 
Abbreviations: $\mathrm{NS}=$ normal saline, $\mathrm{DEX}=$ dexmedetomidine, $\mathrm{T} 0=1$ day before surgery, $\mathrm{T} 1=$ 1 day after surgery, $\mathrm{T} 3=3$ days after surgery, $\mathrm{IL}=$ interleukin, $\mathrm{TNF}=$ tumor necrosis factor.

Table 4. Intraoperative adverse events

\begin{tabular}{llll}
\hline & NS group (N=108) & DEX group (N=110) & P-value \\
\hline Tachycardia & $6(5.56 \%)$ & $10(9.09 \%)$ & 0.317 \\
Bradycardia & $16(14.81 \%)$ & $18(16.36 \%)$ & 0.753 \\
\hline Hypertension & $14(12.96 \%)$ & $22(20.00 \%)$ & 0.162 \\
\hline Hypotension & $8(7.41 \%)$ & $8(7.27 \%)$ & 0.970 \\
\hline
\end{tabular}

Abbreviations: $\mathrm{NS}=$ normal saline, $\mathrm{DEX}=$ dexmedetomidine.

\section{Figures}




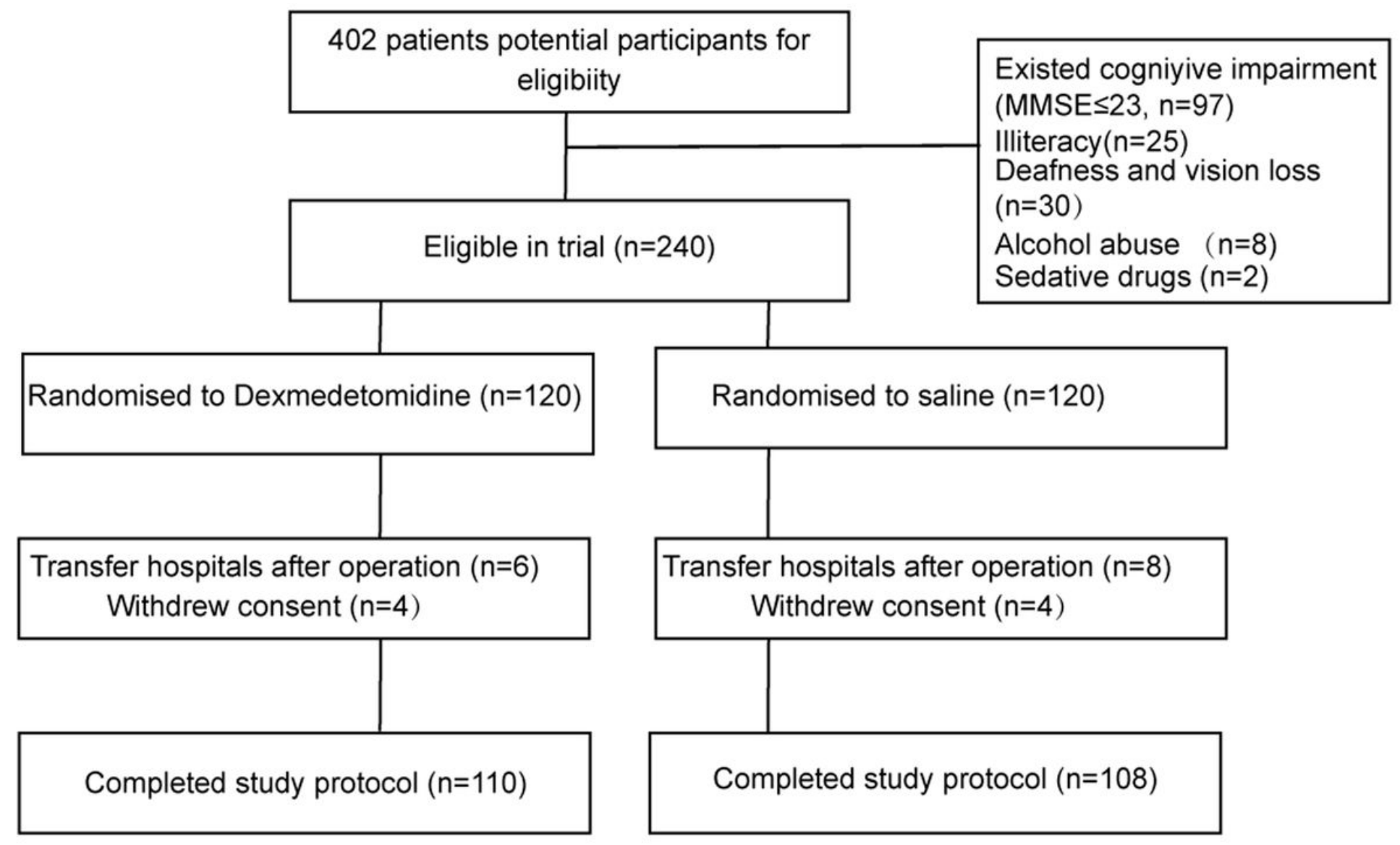

Figure 1

Flow chart of study inclusion, randomization, and follow up. 
A
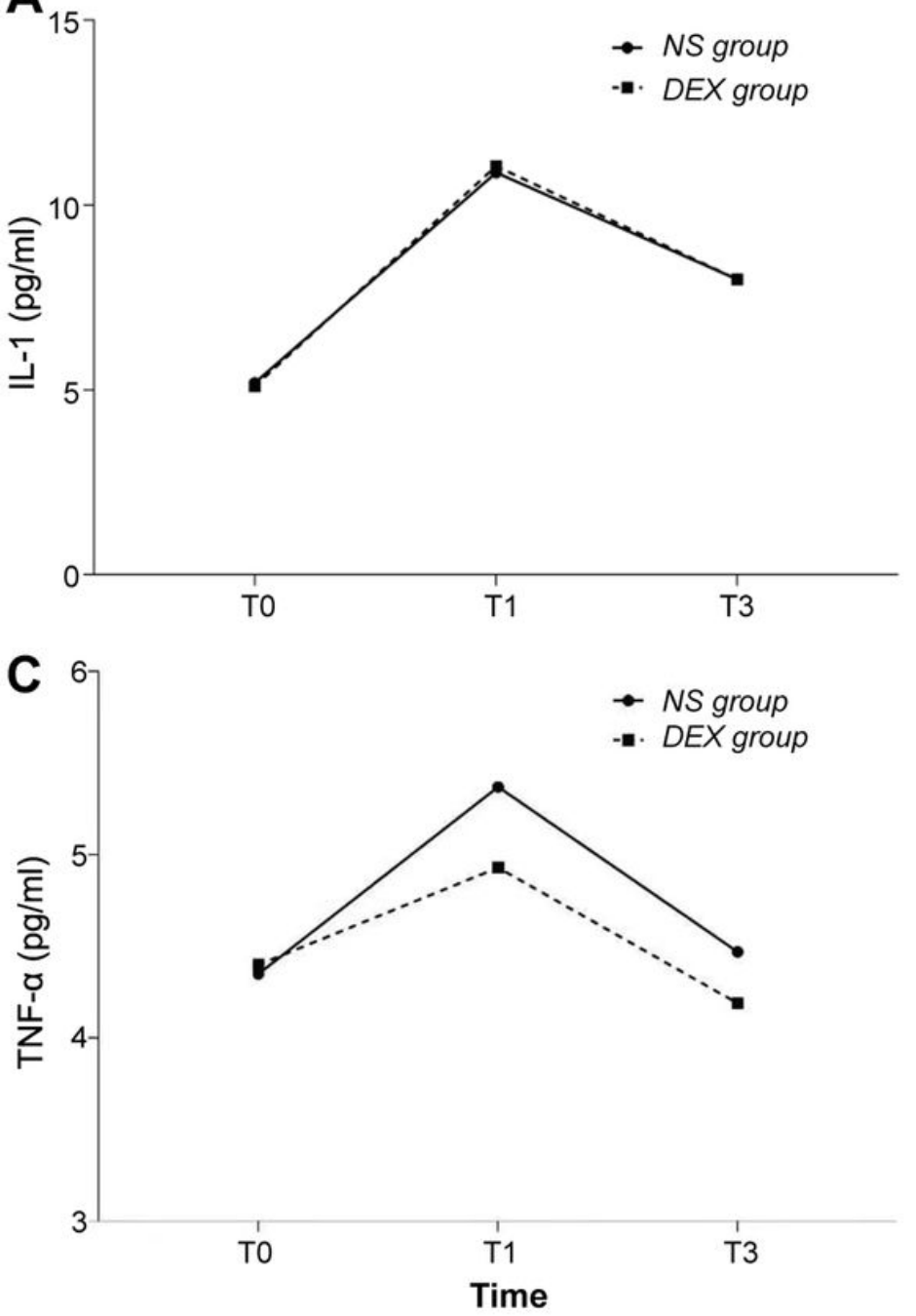

B

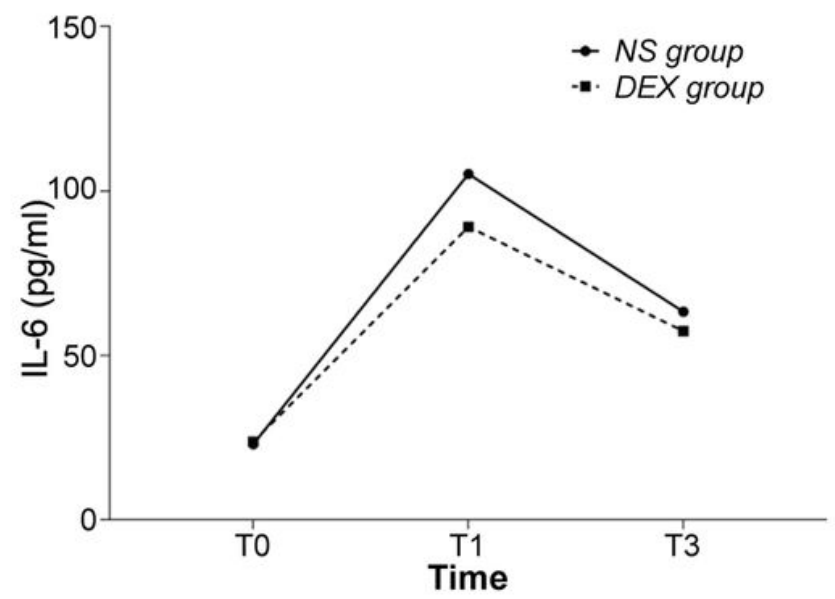

Figure 2

Change in interleukin (IL)-1, IL-6 and tumor necrosis factor (TNF)-a levels over time. 